\title{
PROTÓTIPO DE MONITORAMENTO WI-FI DE UMA INCUBADORA
}

\author{
Eliza Piolli O. Prado*, Katherinne Lima de Abreu Silva** e Vitor Carvalho Morais*** \\ *Universidade Federal de Uberlândia, Uberlândia, Brasil \\ ** Universidade Federal de Uberlândia, Uberlândia, Brasil \\ *** Universidade Federal de Uberlândia, Uberlândia, Brasil \\ e-mail: elizazaaprado@gmail.com, katherinnelima@hotmail.com.br, victorcbmorais@hotmail.com
}

Resumo: O controle de temperatura de Incubadoras
Neo-natais,na maioria das vezes é feito via transmissão
a cabo, e então surge a problemática visto que muitas
vezes essa transmissão é dificultada devido ao fato da
equipe profissional que faz o monitoramento apenas
visualiza esses valores ao entrarem dentro das sala onde
estão as incubadoras, e com esse projeto de
monitoramento via Wi-Fi, a equipe terá uma
visualização até mesmo em outros lugares a não ser a
sala onde as incubadoras estão instaladas, sendo
possível ter uma central de monitoramento separada,
pois a transmissão de dados Wi-Fi possui um alcance de
dezenas de metros.

Palavras-chave: Sensor de temperatura, Wifi, controle de temperatura.

\begin{abstract}
The temperature control of Neo-natal incubators, most of the time is done via transmission cable, and there is the issue where often such communication is difficult due to the fact that the team that is monitoring only see these values to come within the room where the incubators, and how this monitoring project via $W i-F i$, the team will have a view even elsewhere unless the room where incubators are installed, being possible to have a central separate monitoring because Wi-Fi transmission data has a range of tens of meters.
\end{abstract}

Keywords: Temperature sensor, wifi, temperature control.

\section{Introdução}

A incubadora consiste de um ambiente fechado com paredes transparentes, geralmente feitas de acrílico, para permitir a monitoração visual do recém-nascido. Ela possui várias aberturas (portinholas) para manusear o recém-nascido sem a necessidade de abrir sua cúpula.

O recém-nascido é colocado sobre um colchão feito com material macio e isolante térmico, sob este colchão existe uma bandeja de alumínio, sob esta parede metálica que isola o compartimento do recém-nascido do resto do equipamento estão os dispositivos eletromecânicos de controle (aquecimento, umidificação e circulação do ar).

Desta maneira, observa se que é de extrema importância, o controle de temperatura, umidade etc. para que a equipe profissional do hospital possa de maneira clara e precisa monitorar quaisquer variações destes valores e por consequência, ser possível tomar as atitudes cabíveis para que a temperatura do equipamento volte ao valor que se espera.

A princípio o projeto foi apresentado e discutido em sala de aula, na disciplina de Projeto Interdisciplinar, e com o passar das aulas foi se implementando novas ideias que seriam de melhor abordagem para o mesmo. $\mathrm{O}$ trabalho foi evoluindo de maneira rápida, visto que buscou se ajuda de alguns professores no estabelecimento dos módulos do projeto. A motivação da escolha do projeto em si, se baseou na necessidade de um controle rígido de temperatura e unidade em incubadoras, porém a implementação desse método, como a transmissão de dados via Wi-Fi, para maior comodidade da equipe profissional, e para que como o uso do módulo, os dados fossem atualizados em tempo real, o que era mais difícil de ocorrer usando a transmissão a cabo normal.

\section{Materiais e Métodos}

Após, o entendimento da necessidade do preto ser feito e nos benefícios que o mesmo traria para o ambiente hospitalar, a metodologia, se baseou em avanços semanais durante o primeiro semestre letivo de 2016 da Universidade Federal de Uberlândia, na disciplina de Projeto Interdisciplinar, com isso, durante o semestre letivo, foi apresentado semanalmente o desenvolvimento do protótipo, até a verso final do mesmo. Logo, após o orçamento dos materiais que deveriam ser utiliza- dos, partiu se para a compra dos mesmos, e implementação junto a parte de programação do sensor de temperatura e umidade, bem como o módulo.

Decidiu-se então, usar o sensor DHT11, pois este um sensor de temperatura e umidade que permite fazer leituras de temperaturas entre 0 a 50 Celsius e umidade entre 20 a $90 \%$, muito usado para projetos com Arduino. E o Módulo Wireless ESP8266, pois com este foi possível facilmente se conectar a um ponto de acesso Wi-Fi e possui fácil comunicação com o Arduino.

Para entender o funcionamento do sensor, notou-se que era necessário determinar seus terminais, onde tem se apenas 4 pinos : Vcc, Data e GND e um para bit de 


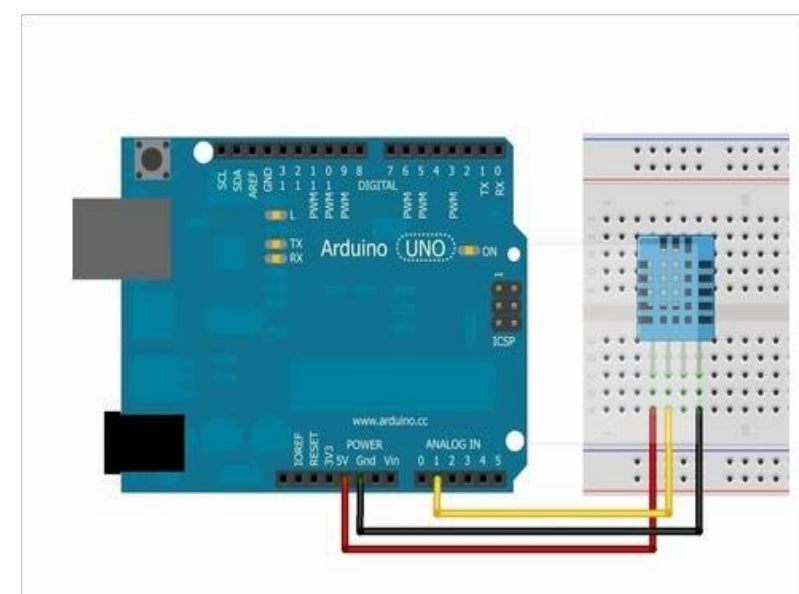

paridade. A conexão deste com o Arduino foi feita utilizando manuais obtidos pela internet. Apenas três pinos são usados: GND, VCC e Dados, o pino de dados do sensor foi conectado ao pino dois do Arduino.

$\mathrm{O}$ interessante neste componente o protocolo usado para transferir dados entre o MCDU (unidade de exibição de controle) e o sensor, pois as leituras do sensor são enviadas usando apenas um único fio de barramento, onde o pino de dados é ligado na porta Digital do Arduino, e os demais na Porta Analógica. Após essa etapa de estudo do sensor, foi possível fazer o estudo do Módulo Wi-Fi, e como este funciona, o interessante neste componente o protocolo usado para transferir dados entre o MCDU (unidade de exibição de controle) e o sensor, pois as leituras do sensor são enviadas usando apena um único fio de barramento, e o pino de dados ligado na porta Digital do Arduino, e os demais na Porta Analógica, o alcance aproximado deste de 91 metros, sendo sua tensão de operação: $3.3 \mathrm{~V}$, e a comunicação serial feita nos pinos TX e RX do Arduino.

Decide-se então utilizar os seguintes componentes para montagem do circuito, que determinamos também usar rede wi-fi.

- $\quad$ Dois sensores de temperatura DHTII;

- Arduino UNO;

- Protoboard 400 pontos;

- Modulo Wireless ESP8266;

- Dois resistores $10 \mathrm{k} \Omega$;

Parte da programação, foi realizada na linguagem C, e fornece os requisitos do programa necessários, para que se possa realizar as leituras de Temperatura e Umidade com isso, durante o semestre letivo, foi apresentado semanalmente o desenvolvimento do protótipo, até a verso final do mesmo.

O protótipo foi pensado para ser implementado em incubadoras neonatais, porém, pode ser utilizado para monitoramento de diversas unidades que precisam de um controle rígido de temperatura e umidade. Para a montagem desse protótipo, foi usado o micro controlador Arduino Uno, bem como os sensores e modulo Wireless, como mostrado na figura abaixo.
A montagem foi bastante simples e para saber como ligar os devidos componentes foi consultado o datasheet dos mesmos. A ligação do sensor de temperatura DHTII foi feita de acordo com a imagem abaixo:

Já o módulo WI-FI ESP8266-01 foi conectado ao arduino como mostrado na figura abaixo:

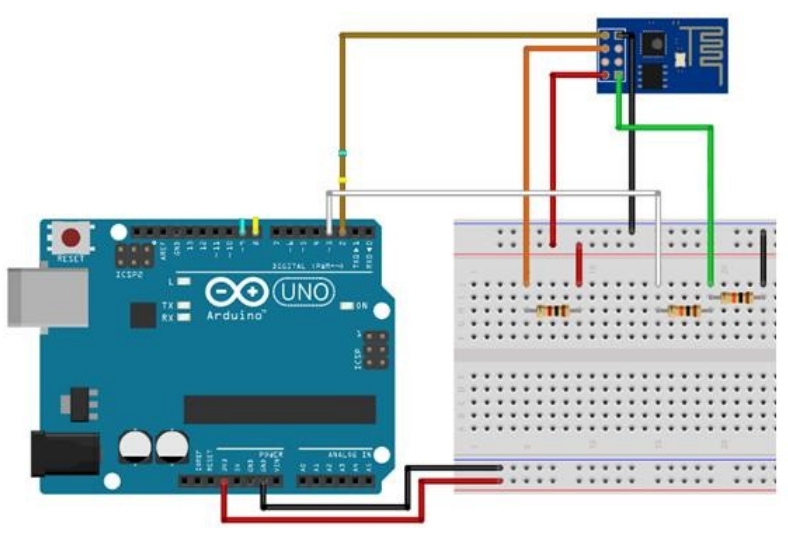

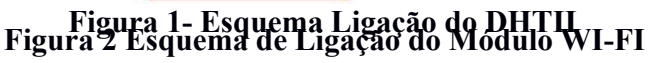

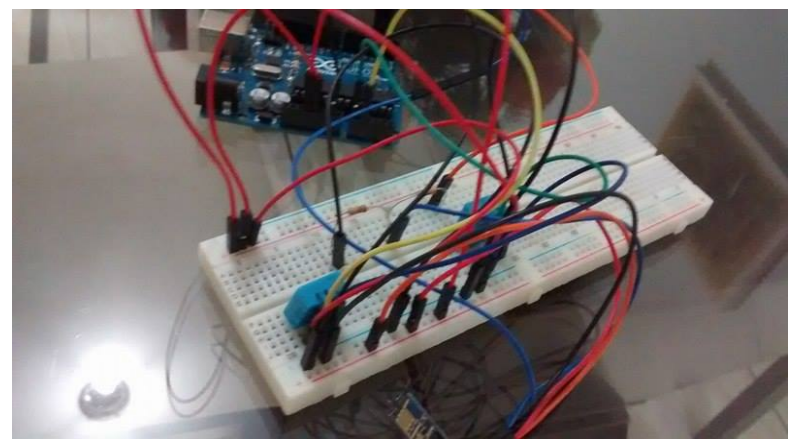

Figura 3 -Protótipo Real do sensor e módulo

\section{Resultados}

Como o esperado era conseguir enviar o sensor de temperatura e umidade via wifi para uma central de monitoramento num raio pré-determinado. $\mathrm{O}$ objetivo do trabalho foi concluído porém para acessa os dados era necessário entrar no ip fornecido pelo modulo wifi através do arduino. Através da imagem abaixo nota-se o resultado final obtido no projeto: 


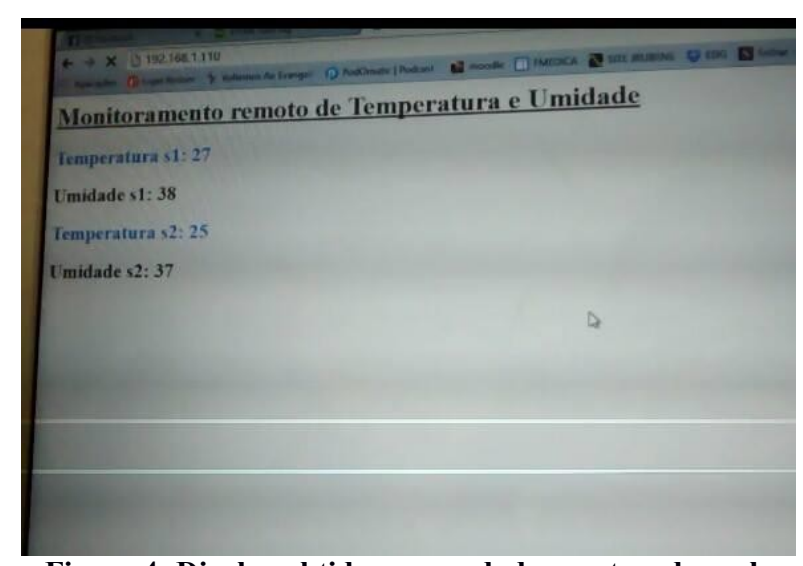

Figura 4- Display obtido com os dados capturados pelo módulo WI-FI.

Na prática, esse projeto poderá funcionar em sincronia com as demais incubadoras, visto que nesse caso foi realizado o protótipo para apenas duas incubadoras, porém na pratica, como na maioria das vezes existem diversas incubadoras na mesma sala, essa medição poderá ser realizada em diversas destas, possibilitando assim bons resultados no ambiente hospitalar.

\section{Discussão}

A discussão do projeto girou em torno da implementação do mesmo, em ambientes neonatais, mesmo que não completamente, que ele trouxesse algo útil, que inspirasse alguma ideia de implementação, nem que seja um monitoramento separado. Decidiu-se então com o intuito de aprimorar o curso e de criar algo inspirador e nos dedicar a esse projeto. Começamos esse projeto porque achamos esse tema um tanto inovador para o meio hospitalar, pois não né muito comum se encontrar monitoramentos como esse, e achamos útil porque isso claramente facilitaria as atividades dos profissionais no âmbito hospitalar, contribuindo para uma melhora nas atividades desse setor.

\section{Conclusão}

Então através do projeto, conclui-se que, após o término do trabalho de que além da importância acadêmica, o mesmo oferece uma grande importância para entendermos o processo de transmissão de dados via rede sem fio, e a exibição desses dados, bem como é obtido o valor de temperatura que se deseja monitorar. Foi de extrema gratificação poder ver o funcionamento detalhado do projeto bem como sua aplicação a nível de demonstração, para os alunos de introdução a eng. biomédica e para os colegas de sala de aula. Foi proveitoso em todos os sentidos, e exigiu que nos preparássemos para a realização de várias etapas as quais não tínhamos facilidade em executar. Seja na parte eletrônica e teórica, quanto no aprendizado e crescimento acadêmico, encontramos que o trabalho foi extremamente importante

\section{Agradecimentos}

Agradecimentos ao Prof. Dr. Adriano de Oliveira Andrade, que nos apoiou na realização do projeto e a Universidade Federal de Uberlândia por ter nos proporcionado estutura físca para poder concluir o mesmo.

\section{Referências}

[1] ASSOCIAÇÃO BRASILEIRA DE NORMAS TÉCNICAS. Equipamento Eletromédico Prescrições particulares para segurança de incubadoras de recémnascidos. NBR IEC 60601-2-19 Emenda 1. Rio de Janeiro, 2000.

[2] N. AGOSTINI. "Sistema Computadorizado para verificação da funcionalidade em incubadoras neonatais". 2003. 79 f. Dissertação(Programa de PósGraduação em Engenharia Elétrica) - Universidade Federal de Santa Catarina, Florianópolis. 2003.

[3] Sensor de Umidade e Temperatura DHTII- Blog FlipFlop. Acessado em maio, Disponível em: http://blog.filipeflop.com/sensores/monitorandotemperatura-e-umidade-com-o-sensor-dht11.html 